Z Herz-Thorax- Gefäßchir 2010 • 24:333-333

DOI 10.1007/s00398-010-0818-6

Online publiziert: 3. Dezember 2010

(c) Springer-Verlag 2010

\author{
Hans-Reinhard Zerkowski \\ Managing Editor HTG, Riehen
}

\section{Alles Gute für eine spannende Zukunft!}

Die Zukunft der Herzchirurgie, die Perspektiventwicklung des Fachs und das Fortkommen unseres Nachwuchses ruhen auf drei, mitunter auch ein wenig schwächelnden, Säulen: Forschung und Innovation, Evidenz in der Krankenversorgung und die Sichtbarmachung von beidem nach innen und außen. Letztere wird schon heute getragen von unseren jungen Kolleginnen und Kollegen, von Ihnen, die Sie zu Recht vehementer denn je fordern, die notwendige Perspektive für die Entwicklung des Fachs zu schaffen. Um im Bild zu bleiben - es ist eben nicht mehr ausreichend, auf den Schultern von Giganten zu stehen und in die Zukunft zu schauen. Man will auch etwas sehen.

Angesichts der Begeisterung für Fach, Fortschritt, tägliche Arbeit, in jüngerer Vergangenheit auch der Konkurrenzsituation zu anderen Fächern, im immer wichtiger werdenden Bestreben, Evidenz zur Richtschnur des eigenen Handelns zum Wohle der uns sich anvertrauenden $\mathrm{Pa}$ tienten zu machen, droht die Veränderung unserer Gesellschaft im eigenen professionellen Umfeld übersehen zu werden. Der mündige, partnerschaftlich perzipierende Patient wurde Realität; dennoch erwartete man wie selbstverständlich noch immer den stets verfügbaren, nie unzufriedenen, genügsamen, sowohl zuwartenden als auch vorwärts strebenden Assistenten meiner chirurgischen Jugend, der längst (völlig zu Recht!) verschwunden ist.

Nicht zuletzt gehört zum Fördern, das dem Fordern wie der Schatten folgen sollte, das Ausbilden. Ausbildung, chirurgische Erziehung (ein altmodisches Wort, das ich bewusst wähle, weil Bildung und Ausbildung mehr sein sollten als Wissens- vermittlung) beinhalten nicht nur, Indikationsstellungen zu vermitteln und Techniken zu trainieren, sondern auch das Vorleben entsprechender Fähigkeiten, Vorbild sein und strukturiert die verschiedenen Facetten des Fachs zusammenbringen. Ein mitunter angesichts des Drucks von Case-mix-Punkten und Arbeitszeitgesetzen schwieriges Unterfangen; Verbesserung war und ist notwendig.

Ein kleiner Baustein im Gesamtprogramm "Verbessert die Weiterbildung" sollte die Zeitschrift für Herz-, Thorax- und Gefäßchirurgie werden. Im Jahr 2007 trafen sich einige an der Weiterbildung unseres Nachwuchses besonders Interessierte mit den Zeitschriftenmachern des Springer Verlags und dem Gründer der HTG, Professor Roland Hetzer, in Hamburg, um etwas Neues zu gestalten: eine Plattform für das Kaleidoskop aus Entwicklungen, Fakten, Strömungen in unserem Fach und um es herum, kurz: eine Orientierungsund Weiterbildungshilfe. Nach Monaten der Projektierung und Planung konnte ich als Geburtshelfer die „neue HTG“ aus der Taufe heben. Spannende Jahre schlossen sich an. Das Herausgeberkollegium agierte gelegentlich als Lehrer, meist als wohlmeinender Pate. Vieles ist uns gut gelungen, wie ich meine. Das Kind lernte laufen, wuchs heran. Es wird wahrgenommen; gemessen am modernen Medium des Artikel-Downloads ist es sogar ausgesprochen beliebt. Insgesamt ist die Verbindung zur Zeitschrift für Herz-, Thorax- und Gefäßchirurgie jedoch eher der eines guten Freundes der Familie vergleichbar; gemessen am Abonnement, an der Verbreitung der Druckversion, die doch so mancher schätzt - in der Pause, unterwegs, zu
Hause - ist die HTG innerhalb der Familie Herzchirurgie noch nicht wirklich angekommen. Vielleicht gelingt dies am Ende eines seit langem begonnenen Prozesses der Gesamtstrukturierung von Wissenschaft, Weiterbildung und Öffentlichkeitsarbeit unserer Fachgesellschaft.

Der immer (und noch) begeisterte Geburtshelfer musste realisieren, dass sich unter dem Druck des Alltags nach mehr als drei Jahren die Wege trennen müssen. Die Zeitschrift für Herz-, Thorax- und Gefäßchirurgie, ein Stück weit sicher meine HTG, wird in der Obhut einer neuen Schriftleitung zweifellos weiter gedeihen, sich fortentwickeln und Ihre HTG bleiben!

Ihnen allen, unseren Lesern, meinen Kollegen, dem Verlag und allen Unterstützern um uns herum, danke ich für eine interessante und befriedigende Zeit.

Ich wünsche Ihnen und Ihrer Zeitschrift für die Zukunft alles Gute. Glück auf!

Ihr

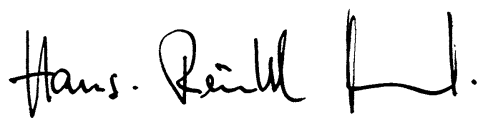

Hans-Reinhard Zerkowski

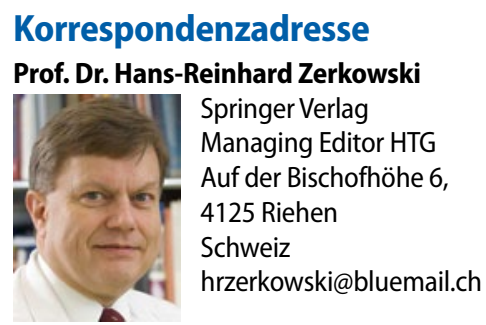

